\title{
PERCEPÇÃO SOBRE AS ESPECIFICIDADES DO CUIDADO COM PACIENTES SUBMETIDOS À HEMODIÁLISE: UM RELATO DE EXPERIÊNCIA
}

\author{
Perception on the specificities of care with patients submitted to \\ hemodialysis: an experience report
}

Guilherme Parreira Vaz ${ }^{1}$, Evandro Leite Bitencourt ${ }^{1,2}$

\begin{abstract}
RESUMO
Quais as implicações existentes no cuidado de pacientes submetidos à hemodiálise? Partindo desse questionamento o objetivo deste artigo é identificar e discutir os principais aspectos relacionados às especificidades do cuidado com pacientes submetidos à hemodiálise. Isso será feito por meio de um relato de experiência a partir da percepção vivenciada por um estudante do 40 período do curso de Medicina da Universidade Federal do Tocantins, campus Palmas. O campo de estágio para a realização do trabalho foi através de uma visita técnica à ala de Nefrologia do Hospital Geral Público de Palmas (HGPP), localizado no município de Palmas, Tocantins, na qual foram feitas a observação, análise e registro das principais impressões obtidas. Os resultados apontam que a prática do cuidar envolve o protagonismo multiprofissional, o qual é influenciado pelo próprio sistema de saúde. Foi evidenciado que a infraestrutura hospitalar representa fator significativo para a ocorrência de quedas, bem como é agente relevante no desenvolvimento de doenças infecciosas. Além disso, a execução inadequada dos serviços em saúde associada à superlotação de leitos compromete a privacidade do assistido. Por fim, o cidadão dialisado necessita do controle alimentar que tem demonstrado resultados significativos para a melhoria de seu bem-estar.
\end{abstract}

Palavras- Chave: Paciente. Cuidado. Hemodiálise.

\begin{abstract}
Citação: Vaz GP, Bitencourt EL. (2020) Percepção sobre as especificidades do cuidado com pacientes submetidos à hemodiálise: um relato de experiência Revista de Patologia do Tocantins, 7(1):.78-82
\end{abstract}

Instituição: ${ }^{1}$ Acadêmico(a) de Medicina, Universidade Federal do Tocantins, Tocantins, Brasil. ${ }^{2}$ Graduado em Química, Mestre em Química, Pesquisador Instituto Médico Legal do Tocantins (IML/TO), Palmas, Brasil.

Autor correspondente: Guilherme Parreira Vaz; parreirameduft@gmail.com

Editor: Guedes V. R. Medicina, Universidade Federal do Tocantins, Brasil.

Publicado: 09 de junho de 2019.

Direitos Autorais: (c) 2020 Vaz et al. Este é um artigo de acesso aberto que permite o uso, a distribuição e a reprodução sem restrições em qualquer meio, desde que o autor original e a fonte sejam creditados. Conflito de interesses: os autores declararam que não existem conflitos de interesses.

\begin{abstract}
What are the implications of caring for patients undergoing hemodialysis? Starting from this questioning the objective of this article is to identify and discuss the main aspects related to the specificities of the care with patients submitted to hemodialysis. This will be done through an experience report from the perception experienced by a student of the fourth period of the Medicine course at the Federal University of Tocantins, Palmas campus. The field of study for the accomplishment of the work was through a technical visit to the Nephrology ward of the General Public Hospital of Palmas (HGPP), located in the municipality of Palmas, Tocantins, in which observation, analysis and recording of the main impressions obtained. The results indicate that the practice of caring involves multiprofessional protagonism, which is influenced by the health system itself. It was evidenced that the hospital infrastructure represents a significant factor for the occurrence of falls, as well as it is a relevant agent in the development of infectious diseases. In addition, the inadequate execution of health services associated with overcrowding of beds compromises the privacy of the assisted. Finally, the dialysed citizen needs food control that has shown significant results to improve his well-being.
\end{abstract}

Keywords: Patient. Caution. Hemodialysis. 


\section{INTRODUÇÃO}

A hemodiálise é um procedimento mecânico que pode ser caracterizado pela filtração sanguínea feita por meio de um capilar. Essa é utilizada para a retirada dos produtos oriundos da degradação do metabolismo e excesso de líquido. Tal tratamento é realizado cerca de 3 vezes por semana, com duração aproximada de 4 horas, sendo que os pacientes necessitam de medicamentos e dieta específica a partir de seu início (MARINHO et al., 2018).

Nesse sentido, o contexto para a adoção dessa conduta médica se justifica pelo desenvolvimento da Doença Renal Crônica (DRC). Essa morbidade tem assumido papel de destaque no Brasil, seja pelo impacto negativo na qualidade de vida do portador, seja pelos custos gerados para o Sistema Único de Saúde (SUS). A partir do desenvolvimento progressivo da falência renal, os cidadãos precisam ser submetidos aos mecanismos dialíticos, na maioria das vezes, enquanto aguardam o transplante renal (BATISTA et al., 2017).

Por sua vez, no Brasil, o número de rins transplantados por ano atende apenas $30 \%$ dos brasileiros que aguardam na lista de espera para a cirurgia. Nesse contexto, a diálise destaca-se por sua importância para a manutenção da sobrevida dos acometidos pela insuficiência renal crônica (IRC), uma vez que é utilizada por cerca de $89,4 \%$ dessa população . Sendo assim, a internação hospitalar primária, que ocorre geralmente durante um período significativo até a transferência para a unidade especializada, é a alternativa mais comum existente no sistema público de saúde a fim de amenizar as complicações fisiológicas decorrentes da patologia renal para o organismo (PESSOA e LINHARES, 2015).

Dessa maneira, a submissão dos portadores de IRC às máquinas dialíticas promove alterações e impactos significativos na qualidade de vida dos mesmos desde as primeiras sessões de tal tratamento. Dentre os principais efeitos destacam-se mudanças relacionadas a aspectos físicos, psicológicos e sociais do indivíduo, refletindo na dinâmica das relações familiares. Isso pode ser explicado a partir da análise do próprio processo de hemodiálise, visto que por ser contínuo, no decorrer do tempo pode favorecer a modificações na imagem corporal, dependência, restrições dietéticas e hídricas, bem como isolamento social do usuário (TAKEMOTO, 2011).

Além disso, devido às diversas consequências enfrentadas pelo cidadão ao ser dialisado, faz-se necessária a adoção de uma série de cuidados específicos para esse grupo de pessoas, tendo em vista que são bastante comuns as intercorrências clínicas. Essas muitas vezes podem levar ao óbito do paciente. Sendo assim, o ato de cuidar envolve várias vertentes que se somam em prol da atenção prestada ao enfermo, no intuito de se promover um atendimento humanizado e eficaz que não se limita ao tecnicismo executado por milhares de profissionais de saúde (RODRIGUES e BOTTI, 2009).

É válido ressaltar ainda, que se tratando de um grupo tão singular, muitas vezes o automatismo em se cuidar de pessoas crônicas traz consigo o "fazer por fazer", ignorando nesse contexto a complexidade que está por trás do simples apertar o botão da máquina dialítica. Essa realidade é vivenciada em muitas unidades de atendimento especializado, prejudicando consideravelmente o bem-estar daqueles que precisam de apoio e do verdadeiro cuidado em saúde (WILLIG et al., 2006).

Nessa perspectiva, o objetivo do presente artigo é identificar e discutir os principais aspectos relacionados às especificidades do cuidado com pacientes submetidos à hemodiálise. Isso será feito por meio de um relato de experiência a partir da percepção vivenciada por um estudante do 40 período do curso de Medicina da Universidade Federal do Tocantins, campus Palmas. O campo de estágio prático para a realização do trabalho foi através de uma visita técnica à ala de Nefrologia do Hospital Geral Público de Palmas (HGPP), localizado no município de Palmas, Tocantins, na qual foram feitas as atividades de observação, análise e registro das principais impressões obtidas.

\section{METODOLOGIA}

O tipo de estudo utilizado para essa produção científica foi descritivo, feito a partir dos relatos de experiência de um estudante do 4으o período do curso de Medicina da Universidade Federal do Tocantins, campus Palmas, que realizou uma visita técnica durante aproximadamente 30 dias na Ala de Nefrologia do HGPP, no segundo semestre de 2018.

O discente foi orientado pela professora responsável desde o início da atividade, que passou pela aprovação inicial do Núcleo de Educação Permanente (NEP) do HGPP. Essa ação contou como instrumento metodológico a observação, o diálogo e registro de informações a partir da autorização prévia dos profissionais de saúde, funcionários do setor hospitalar alvo da vivência.

Além disso, a percepção subjetiva do graduando a cerca das características a serem analisadas ao longo do período de estágio também foram descritas diariamente com o auxílio de um roteiro de aula prática. Esse foi elaborado pelo docente responsável pela visita técnica, em parceria com o aluno protagonista, que juntos tentaram abranger não só diversos aspectos do cuidado necessário para com o paciente em hemodiálise, mas também sobre condições de infraestrutura e funcionamento do setor de Nefrologia no estabelecimento.

Nesse sentido, inicialmente foi necessário o preenchimento de uma solicitação formal de visita técnica a ser entregue ao NEP do hospital. Nesta constavam, dentre outras informações, os dados pessoas do universitário, os objetivos gerais da ação, o docente responsável pelo aluno, assim como o período e dias de permanência do acadêmico no campo de prática. Na figura abaixo se destaca o formulário para a visita técnica, obrigatório perante o Núcleo Permanente de Educação (NEP) do HGPP, e utilizado para o cumprimento da atividade prática em saúde.

Figura 1- Formulário de visita técnica. 
Solicitacão de Visita Técnica Pessoa Física

\begin{tabular}{|c|c|c|}
\hline \multirow{2}{*}{\multicolumn{3}{|c|}{$\begin{array}{l}\text { Solicitação de Visita Técnica (Pessoa Fisica) N } \\
\text { Nome da Unidade ou Hospital: Hospital Geral Público de Palmas (HGPP) } \\
\text { Dados Pessoais do Solicitante }\end{array}$}} \\
\hline & & \\
\hline \multicolumn{3}{|c|}{\begin{tabular}{l|l} 
NOME: & Data de Nasc:
\end{tabular}} \\
\hline \multicolumn{2}{|l|}{ Profissão: } & $\mathrm{N}^{\circ}$ do Conselho Profissional: - \\
\hline \multicolumn{2}{|l|}{$\mathrm{N}^{\circ} \mathrm{RG}:$} & $\mathrm{N}^{\circ} \mathrm{CPF}:$ \\
\hline \multicolumn{3}{|l|}{ Endereço: } \\
\hline \multirow[t]{2}{*}{ Bairro: } & CEP: & Cidade: \\
\hline & Celular: & E-mail: \\
\hline \multicolumn{3}{|l|}{ Escolaridade: } \\
\hline \multicolumn{3}{|l|}{ Escola de Formacão: } \\
\hline \multicolumn{3}{|c|}{ Nome do Curso: } \\
\hline \multirow{2}{*}{\multicolumn{3}{|c|}{$\begin{array}{l}\text { Ano do Inicio: } \\
\text { Curso de Especializacão: - }\end{array}$}} \\
\hline & & \\
\hline \\
\hline Mês/Ano de Inicio:- & & Mês/Ano do Término:- \\
\hline \multicolumn{3}{|c|}{ Área de Interesse da Visita } \\
\hline \multicolumn{3}{|c|}{ Data da Visita: } \\
\hline \multirow{2}{*}{\multicolumn{3}{|c|}{ Período: Manhã ( ) Tarde ( ) Noite ( ) }} \\
\hline & & Objetivo da Visita: \\
\hline \multirow{2}{*}{\multicolumn{3}{|c|}{\begin{tabular}{l|l} 
Data: & Assinatura do Solicitante \\
Manifestação do Núcleo de Educação Permanente
\end{tabular}}} \\
\hline & & \\
\hline \multicolumn{3}{|c|}{ Parecer: ( ) Aprovado } \\
\hline \multicolumn{3}{|c|}{ Não Aprovado ( ) Justificativa: } \\
\hline \multirow{2}{*}{\multicolumn{3}{|c|}{\begin{tabular}{ll|l} 
Encaminhado para: & Data: \\
& Assinatura e Carimbo do NEP
\end{tabular}}} \\
\hline Assinatura e Carimbo do NEP & & \\
\hline \multicolumn{3}{|c|}{ Manifestaçäo da Área ou Setor Solicitado } \\
\hline \multicolumn{3}{|c|}{ Parecer: ( ) Aprovado } \\
\hline \multicolumn{3}{|c|}{ Não Aprovado ( ) Justificativa: } \\
\hline Encaminhado para: & & Data: \\
\hline
\end{tabular}

Sendo assim, após a aprovação do requerimento pelas autoridades responsáveis pelo NEP, foi feita a confecção e entrega de um crachá para o aluno. Esse documento concedia a permissão temporária ao estudante para frequentar as dependências hospitalares do HGPP vinculadas à área de Nefrologia

Dessa forma, as vivências na unidade hospitalar tiveram início no dia 15/11/2018 e se encerraram no dia $08 / 12 / 2018$, sendo realizadas sempre às quintas-feiras e sábados de cada semana ininterruptamente. Os horários para a realização das visitas foram estabelecidos a partir da disponibilidade do discente, tendo em vista a grade curricular do mesmo e perfazendo um total de 20 horas ao longo dos 30 dias no local.

\section{RESULTADOS E DISCUSSÃO}

\section{O reconhecimento do campo de prática em saúde}

O primeiro contato do graduando, seja em qualquer curso da área de saúde, com o ambiente hospitalar é cercado por subjetividades e singulares de cada um. A convivência com a doença, a dor e a angústia do outro lado a lado, mediante a todo um conjunto de protocolos a serem seguidos, pode desconstruir conceitos e visões idealizadas relacionadas à própria atuação do profissional em formação. Nesse sentido, seu papel junto à sociedade, bem como o plano de ensino ofertado ao discente passa a ser questionado por ele mesmo fazendo com que educadores e estudantes reflitam sobre a qualidade do conhecimento que estão transmitindo e absolvendo respectivamente (PERBONE e CARVALHO, 2011).

Nesse sentido, inicialmente nos primeiros dias de vivência, o estudante teve a oportunidade de conhecer melhor o funcionamento do Hospital Geral Público de Palmas com ênfase na Ala de Nefrologia, onde esteve por grande parte do período de visita técnica. Foi possível identificar a disposição das especialidades médicas, que são alojadas em 4 (quatro andares) no estabelecimento. Além disso, houve a percepção de diferenças significativas quanto à higiene e organização dos equipamentos utilizados em cada uma das subdivisões de leitos.

Por sua vez, a observação inicial feita no bloco de quartos destinados aos pacientes submetidos à hemodiálise, situado no quarto andar, demonstrou grande disparidade em relação a outras enfermarias. Isso ficou evidenciado, sobretudo em relação à estrutura física do local, que é mais arejada e limpa, permitindo a livre circulação dos cidadãos e funcionários no setor.

Através do diálogo feito com enfermeiros e médicos plantonistas obtiveram-se informações relevantes a cerca do recinto destinado às atividades da Ala "N", denominação genérica dada pela administração do hospital. $O$ ambiente possui 14 leitos em funcionamento, bem como 2 extra. Todos apresentam banheiro, camas, ar condicionado, assim como cadeiras para acomodação dos acompanhantes.

Além disso, foi possível identificar uma sala destinada à permanência do médico plantonista e outra repartição específica para os profissionais da enfermagem. Outro ponto importante refere-se à análise do quadro de funcionários responsáveis pela Ala de Nefrologia. Existem atualmente apenas dois médicos nefrologistas que respondem pelas intercorrências ocorridas no setor, sendo que esses não ficam de modo permanente no local, mas atendem por meio de solicitações feitas pelo médico plantonista ou da equipe de enfermagem.

Essa é composta por 1 (um) enfermeiro e 3 (três) técnicos de enfermagem, que atuam em sistema de rotação, de modo semelhante aos 4 (quatro) médicos clínicos gerais que trabalham em regime de plantão. Há a presença de uma equipe multiprofissional, a qual é composta por 1( um) fisioterapeuta, 1 (um) nutricionista, 1 (um) odontólogo, 1 (um) psicólogo e 1 (um) assistente social.

\section{A Segurança física do paciente renal crônico no ambiente hospitalar}

A temática relacionada à segurança do paciente em hospitais e centros de saúde representa um sério problema de saúde pública que afeta diversos países. Nesse sentido, os riscos os quais comprometem o bem estar dos indivíduos durante sua internação ou passagem por esses estabelecimentos são diversos. Nessa perspectiva, os incidentes que vem ocorrendo decorrentes dessa situação aumentaram significativamente nas últimas décadas e preocupam órgãos de regulamentação pública da saúde (SILVA et al.,2016).

Sendo assim, no ambiente destinado à Ala de Nefrologia do HGPP, foi possível observar características locais que influenciam consideravelmente no bem-estar de seus usuários. Dentre elas, a falta de segurança dentro dos próprios leitos é uma realidade que chamou a atenção. As camas, por exemplo, não possuem dispositivos de segurança em suas extremidades laterais, o que contribui para o aumento do risco de quedas. Isso se justifica tendo em vista as alterações fisiológicas apresentadas pelos pacientes após o termino da hemodiálise, muitos se queixando de tonteiras e enjoos.

Além disso, os cidadãos internados circulam livremente pelos corredores do setor, grande parte das vezes sem a presença de seus acompanhantes. Dessa maneira, sujeitam-se a diversas ocorrências como o piso molhado, macas e cadeiras espalhadas pelo ambiente, bem como a 
circulação de demais profissionais locais constantemente. Outro aspecto a ser considerado é a respeito da manutenção dos refrigeradores. Foi observada a presença de goteiras frequentes nos quartos, uma vez que todos são equipados com ar-condicionado, esses estavam com defeitos na coleta de água, a qual se espalhava pelos leitos.

Por conseguinte, o chão escorregadio associado à humidade aumentada evidencia um ambiente propício para casos de escorregamentos e doenças respiratórias, a começar por gripes e resfriados indesejáveis. Vale ressaltar ainda que embora exista uma equipe de manutenção estrutural dos prédios, falta à frequência de notificação dos danos por parte da própria administração da enfermaria responsável pela especialidade, representando um típico caso de falta da intersetorialidade nos serviços de saúde.

\section{0 paciente em hemodiálise e as infecções hospitalares}

Infecção hospitalar (IH) pode ser definida como aquela adquirida após a internação do paciente e que se manifesta durante e depois da alta do mesmo. A maioria delas manifesta-se a partir de complicações ocorridas em pacientes cujo estado de saúde é grave, os quais são submetidos a tratamentos invasivos de forma correta ou incorretamente. Algumas IH são evitáveis e outras não. As infecções preveníveis caracterizam-se por serem passíveis de intervenção na cadeia de transmissão dos microrganismos enquanto as outras não o são (PEREIRA, et al., 2005).

Outrossim, as ações de controle e prevenção das $\mathrm{IH}$ precisam ser executadas por meio de uma prática articulada de vigilância à saúde. Essa é dependente de ações integradas no âmbito municipal, estadual e federal. É válido ressaltar que uma atuação multiprofissional torna-se indispensável para se determinar a eficácia desta tarefa, uma vez que exige o trabalho em equipe, através de mobilizações participativas e de gestão. Sem a reflexão de ações cotidianas e o reconhecimento da necessidade de mudança, infelizmente, as perspectivas podem ser as piores possíveis (AZAMBUJA et al., 2004).

Por outro lado, retomando a discussão, identificou-se que no setor de Nefrologia o procedimento de higienização periódica dos cateteres, atualmente, ocorre dia sim e dia não, de modo que os profissionais da enfermagem realizam a limpeza e troca de curativos nos pacientes. Essa realidade não era dessa forma há certo tempo, visto que segundo relatos de funcionários locais, após a adoção do protocolo médico o qual determina a obrigatoriedade da higienização dia sim e outro não, ocorreu uma queda expressiva na ocorrência de infecções hospitalares em $30 \%$.

É bem verdade também que a existência dessas não deixou de estar presente, uma vez que são registrados casos de infecções urinárias, assim como outras de menor gravidade significativamente. Um dado relevante é a falta frequente de medicamentos no estabelecimento, como a furosemida, por exemplo. Tal situação contribui para complicações e dificuldade na realização do tratamento dos pacientes RC, que muitas vezes, necessitam comprar com seu próprio dinheiro os fármacos necessários para sua terapia.

Um aspecto interessante a ser discutido, dentro do contexto de morbidades infecciosas, é sobre o ambiente destinado à realização de procedimentos específicos da especialidade médica em questão. De acordo com os médicos servidores do departamento não existe uma sala exclusiva para a inserção de cateteres, por exemplo, ou remoção do mesmo. Tal fato desperta a reflexão a cerca do risco de contaminação dentro dos leitos, uma vez que são dois cidadãos internados por quarto e durante a execução de qualquer intervenção médica em um, o outro na maioria das vezes vivencia diretamente ou indiretamente o ocorrido.

Além disso, a existência de apenas duas máquinas de hemodiálise para atender a todas as enfermarias dependentes de serviços nefrológicos representa um sério problema de saúde pública que afeta o Hospital Geral Público de Palmas. Foi possível perceber complicações frequentes em pacientes decorrentes da falta de diálise dentro do tempo adequado para cada quadro clinico. Dessa maneira afirma-se que existe um grande conflito entre a necessidade de se fazer mais pelo assistido e a ausência de recursos por parte do sistema de saúde.

Outro fator que potencializa a ocorrência de infecções hospitalares no setor de hemodiálise é o tempo de internação dos cidadãos. O município de Palmas atualmente não possui uma clínica pública para a realização periódica do procedimento dialítico. Sendo assim, a cidade conta com um estabelecimento privado que presta serviços para a secretaria de saúde, oferecendo uma quantidade limitada de leitos para aqueles que farão continuamente o uso desse tratamento. Por tudo isso, geralmente até serem encaminhados para esse local os doentes ficam cerca de 4 a 5 meses na enfermaria do HGPP.

\section{Quanto à privacidade do paciente dialisado em ambiente hospitalar}

Tratar sobre a privacidade é, de fato, um assunto bastante complexo o qual predispõe várias linhas de discussão e debates. Todavia, no presente contexto analisado, sua abordagem mostrou-se necessária por alguns motivos particulares. O tratamento hemodialítico é caracterizado por sua vertente de caráter físico, biológico e orgânico para a manutenção das funções vitais do individuo assistido (MOURA et al, 2015).

Dessa maneira, o uso da tecnologia e de ações técnicas visando à integridade do cidadão configura um aspecto essencial para a funcionalidade do cuidado. Porém, é verdade também que muitas vezes se esquece do sentido humano e relacional implicado no todo. Por isso, a conduta da equipe multiprofissional envolvida pode acabar por ferir a própria privacidade do internado, fazendo-o vivenciar situações desagradáveis e evitáveis por parte dos trabalhadores responsáveis pelo setor (MOURA et al, 2015).

Assim, percebeu-se desde os primeiros dias de vivencia que a disposição dos leitos da Ala "N" representa fator invasivo à privacidade dos usuários. Isso foi constatado ao verificar a lotação de dois pacientes por quarto comprometia o mínimo de liberdade concedida tanto a qualquer um dos doentes quanto a seus acompanhantes. Os quartos por si só já possuem tamanho reduzido, representando uma adaptação feita para a especialidade e não um ambiente previamente planejado para a mesma.

Por conseguinte, deve-se analisar ainda o contexto cultural envolvido especificamente nesta enfermaria. Lá se verificou a presença de cidadãos indígenas os quais dividiam espaço com os demais internados em um mesmo quarto. 
Inclusive, uma dessas pessoas relatou queixas sobre o gestão do setor e atitudes dos funcionários. Por sua vez, a comportamento de um indivíduo oriundo da tribo Xerente. privacidade individual dos assistidos faz-se comprometida Para a colega de quarto, esse estaria chamando por sua aldeia tanto por condutas dos profissionais locais quanto por em voz alta após a meia noite todos os dias. Ademais, a aspectos estruturais do prédio utilizado. percepção a cerca da dificuldade tida por parte da equipe de enfermagem e médica para lidar com este público em especifico foi notável.

Por fim, concluiu-se que a ausência de cadeiras de rodas suficientes para os habitantes temporários da Ala " $N$ " demonstrou ser fator relevante não só para a privacidade quanto para a autonomia e segurança dos assistidos. Dessa forma a reflexão sobre os diversos eventos e particularidades envolvidas no atendimento em hemodiálise revela dados e informações muito significativas que refletem o panorama da saúde pública brasileira.

\section{Restrições alimentares no contexto da Hemodiálise}

Os portadores de IRC apresentam uma dieta restrita em razão das mudanças bioquímicas eminentes. Dentre elas pode ser citado o desequilíbrio mineral, que acarreta níveis elevados de sódio e potássio no organismo. Dessa forma, a educação alimentar e a permanência de hábitos saudáveis de consumo alimentício durante o tratamento dialítico é uma ação muito complexa e fundamental (NASCIMENTO, et al.,2016).

No HGPP, em diversas especialidades médicas nas quais foi observada a atuação da Nefrologia, constatou-se uma rigorosidade notável quanto à alimentação fornecida aos pacientes que sofrem de distúrbios nefrológicos. Dessa maneira, a proibição da presença de filtros de água potável na enfermaria da Ala " $N$ " despertou a atenção evidenciando um cuidado relevante, uma vez que os doentes circulam livremente pelos corredores.

Além disso, o fornecimento regular de refeições balanceadas e controladas pela profissional nutricionista, integrante da equipe multiprofissional do setor, contribui significativamente para a prevenção a distúrbios alimentares. Dentre as precauções observadas destacam-se o cuidado com a quantidade de sal e açúcar presentes nas refeições, tendo em vista a grande frequência de hipertensos e diabéticos que desenvolvem síndromes nefrológicas, assim como outras morbidades associadas.

Outro aspecto importante é a grande variedade de alimentos os quais compõem a dieta semanal dos internados. Esse fator é relevante, visto que impacta consideravelmente no prognóstico dos assistidos. Dessa forma, a gestão integrada da nutrição aliada à odontologia, bem como a frequência diária de um médico responsável pelo setor trouxe resultados positivos para o cuidado eficaz dos pacientes, visto sob a ótica da humanização e integralidade nos serviços de saúde.

\section{CONCLUSÃO}

Identificou-se que a infraestrutura hospitalar contribui para um ambiente propicio a ocorrência de quedas e, consequentemente, causa de acidentes envolvendo os cidadãos internados. Além disso, a percepção a cerca da disposição dos leitos e funcionamento da Ala de Nefrologia revelou a predisposição a infecções hospitalares indesejáveis e que poderiam ser evitadas por meio de mudanças vinculadas à
Já a análise do contexto alimentar demonstrou pontos positivos, destacando-se a seriedade observada quanto à distribuição das refeições e cuidado para se evitar a presença de água consumível. Sendo assim, a atividade de diálogo, observação e registro de informações durante as vivencias permitiu uma análise crítica e real sobre o ambiente de saúde, sobretudo, no contexto hospitalar da Nefrologia.

\section{REFERÊNCIAS BIBLIOGRÁFICAS}

1. AZAMBUJA, E.P. et al. Prevenção e Controle da Infecção Hospitalar: As interfaces com o processo de formação do trabalhador. Revista Texto e Contexto. Vol. 13: p.79-86, 2004.

2. BATISTA, C.M et al. Perfil epidemiológico dos pacientes em lista de espera para o transplante renal. Revista Acta Paul de Enfermagem. Vol. 30(3): p.280-6, 2017.

3. MARINHO, C.L et al. Associação entre características sociodemográficas e qualidade de vida de pacientes renais crônicos em hemodiálise. Revista Cuidarte. Vol. 1, 2018.

4. MOURA, C.M et al. Preservação da privacidade em hemodiálise: percepção dos enfermeiros. Revista de Enfermagem Referência. Vol. 4: p.97-104, 2015.

5. PERBONE, J.G. CARVALHO, E.C. Sentimentos do estudante de enfermagem em seu primeiro contato com pacientes. Revista Brasileira de Enfermagem. Vol. 64(2), 2011.

6. PEREIRA, M.S et al., A infecção hospitalar e suas implicações para o cuidar em enfermagem. Revista Texto e Contexto. Vol. 14(2): p. 250-7, 2005.

7. PESSOA, N.R e LINHARES, F.M. Pacientes em hemodiálise com fístula arteriovenosa: conhecimento, atitude e prática. Escola Anna Nery Revista de Enfermagem. Vol. 19(1). Jan-Mar, 2015.

8. RODRIGUES, T.A. BOTTI, N.C. Cuidar e o ser cuidado na hemodiálise. Revista Acta Paul de Enfermagem. Vol. 22(Especial-Nefrologia): p.528-30, 2009.

9. SILVA, A.C et al. A segurança do paciente em âmbito hospitalar: revisão integrativa da literatura. Revista Cogitare Enfermagem. 2016 v. 21 n. esp: 01-09

10. TAKEMOTO, A. YOKUBO, P.; BEDENDO, J. CARREIRA, L. Avaliação da qualidade de vida em idosos submetidos ao tratamento hemodialítico. Revista Gaúcha de Enfermagem. Vol. 32(2): p. 256-62, 2011.

11. WILLIG, M.H. Gerenciamento e Cuidado em Unidades de Hemodiálise. Revista Brasileira de Enfermagem. Vol. 59(2): p.177-82, 2006 